\title{
Determinantes Sociais da Saúde no pré-natal: um olhar multiprofissional na Atenção
}

\section{Primária}

\author{
Social Determinants of Health in prenatal care: a multidisciplinary view in Primary Health Care \\ Determinantes sociales en la atención prenatal: una mirada multidisciplinar en Atención Primaria \\ de la salud
}

Recebido: 01/03/2021 | Revisado: 08/03/2021 | Aceito: 17/03/2021 | Publicado: 23/03/2021

\author{
Carolina Gabriele Gomes da Rocha \\ ORCID: https://orcid.org/0000-0003-4150-9332 \\ Secretaria Estadual de Saúde de Santa Catarina, Brasil \\ E-mail:carolinagabriele.r@gmail.com \\ Ivonete Teresinha Schülter Buss Heidemann \\ ORCID: https://orcid.org/0000-0001-6216-1633 \\ Universidade Federal de Santa Catarina, Brasil \\ E-mail: ivoneteheideman@gmail.com \\ Jeane Barros de Souza \\ ORCID: https://orcid.org/0000-0002-0512-9765 \\ Universidade Federal da Fronteira Sul, Brasil \\ E-mail: jeane.souza@uff.edu.br \\ Michelle Kuntz Durand \\ ORCID: https://orcid.org/0000-0003-3660-6859 \\ Universidade Federal de Santa Catarina, Brasil \\ E-mail: michakd@hotmail.com \\ Kamila Soares Maciel \\ ORCID: https://orcid.org/0000-0002-0793-1508 \\ Universidade Federal de Santa Catarina, Brasil \\ E-mail:kmaciel153@gmail.com \\ Laura Tais Loureiro Simas \\ ORCID: https://orcid.org/0000-0002-9052-7740 \\ Prefeitura Municipal de Santa María, Brasil \\ E-mail: lauratais.ls@gmail.com
}

\begin{abstract}
Resumo
Objetivo: compreender como os Determinantes Sociais da Saúde são trabalhados pela equipe multiprofissional durante o pré-natal na Atenção Primária à Saúde. Método: estudo qualitativo, tipo ação participante, fundamentado nos preceitos teórico-metodológicos de Paulo Freire. Participaram 40 profissionais da Atenção Primária de um município do Sul do Brasil. A investigação temática aconteceu entre julho e outubro de 2019 e o desvelamento foi conduzido com base na metodologia Freireana, que prevê o processo analítico. Resultados: os participantes evidenciaram que trabalham os determinantes sociais no pré-natal, apesar das barreiras, como demanda excessiva e a falta de recursos humanos. Destacam que o acolhimento, empatia, escuta qualificada e o trabalho em equipe ampliam o olhar sobre os determinantes sociais e propicia um cuidado de qualidade as gestantes. Conclusão: os determinantes sociais são um caminho para o alcance da equidade no pré-natal e consequentemente para melhoria da qualidade ofertada as gestantes na Atenção Primária.

Palavras-chave: Determinantes sociais da saúde; Promoção da saúde; Atenção primária à saúde; Cuidado pré-natal; Equipe de assistência ao paciente.

Abstract

Objective: To understand how the Social Determinants of Health are worked by a multidisciplinary team during prenatal care in Primary Health Care. Method: a qualitative study, type participant action, based on the theoretical and methodological precepts of Paulo Freire. Forty Primary Care professionals from a municipality in the south of Brazil participated. The thematic investigation took place between July and October 2019 was unveiling conducted based on the Freirean methodology, which provides for the analytical process. Results: the participants showed that they work with social determinants in prenatal care, despite the barriers, such as excessive demand and the lack of human resources. They emphasize that welcoming, empathy, qualified listening and teamwork increase the view on social determinants and provide quality care as pregnant women. Conclusion: social determinants are a way to achieve equity in prenatal care and, consequently, to improve the quality offered to pregnant women in Primary Care.
\end{abstract}

Keywords: Social determinants of health; Health promotion; Primary health care; Prenatal care; Patient care team. 


\section{Resumen}

Objetivo: Comprender como los Determinantes Sociales de la Salud son trabajados por un equipo multidisciplinario durante la atención prenatal en Atención Primaria de Salud. Método: estudio cualitativo, tipo acción participante, basado en los preceptos teóricos y metodológicos de Paulo Freire. Participaron cuarenta profesionales de Atención Primaria de un municipio del sur de Brasil. La investigación temática que tuvo lugar entre julio y octubre de 2019 fue develada realizada con base en la metodología freireana, que prevé el proceso analítico. Resultados: las participantes demostraron que trabajan con determinantes sociales en la atención prenatal, a pesar de las barreras, como la excesiva demanda y la falta de recursos humanos. Destacan que la acogida, la empatía, la escucha calificada y el trabajo en equipo aumentan la visión de los determinantes sociales y brindan una atención de calidad como mujeres embarazadas. Conclusión: los determinantes sociales son una vía para lograr la equidad en la atención prenatal y, en consecuencia, para mejorar la calidad ofrecida a las gestantes en Atención Primaria.

Palabras clave: Determinantes sociales de la salud; Promoción de la salud; Atención primaria de salud; Atención prenatal; Grupo de atención al paciente.

\section{Introdução}

Em 1986 acontecia no Brasil a VIII Conferência Nacional de Saúde. A nível internacional, fortalecia-se o movimento moderno de Promoção da Saúde (PS), impulsionando a realização da I Conferência Internacional sobre Promoção da Saúde em Ottawa, Canadá, que resultou na aprovação da Carta de Ottawa e reafirmação deste movimento e a influência dos aspectos sociais sobre a saúde dos indivíduos e da população (World Health Organization, 1986; Heidemann et al., 2006).

$\mathrm{Na}$ Carta de Ottawa foram conceituadas cinco estratégias fundamentais para o alcance da qualidade de vida: a implementação de políticas saudáveis, criação de ambientes favoráveis à saúde, reorientação dos serviços de saúde, reforço da ação comunitária e desenvolvimento de habilidades pessoais. Tais estratégias, articuladas com os Determinantes Sociais da Saúde (DSS), constituem as práticas de PS e proporcionam acesso à informação, ampliando as experiências e habilidades na vida dos sujeitos, tal como apontam oportunidades para os indivíduos fazerem escolhas por uma vida mais saudável (World Health Organization, 1986; Costa et al., 2019).

A Carta de Ottawa reafirma a relevância dos DSS ao ressaltar que se o tratamento das doenças é uma atribuição específica do setor saúde, a PS abarca uma complexidade que o extrapola, necessitando de diversas ações intersetoriais para a sua construção, o que a caracteriza como uma estratégia genuinamente intersetorial (Pinheiro et al., 2015).

A integração da Promoção da Saúde e dos DSS é um desafio, uma vez que o foco no estilo de vida individual continua a ser hegemônico nos serviços de saúde. As ações de PS são geralmente limitadas a identificar os efeitos prejudiciais de determinados comportamentos de vida, atuando na normatização de estilos de vida, com manutenção no foco na doença e na eliminação de hábitos considerados de risco (Heidemann et al., 2018).

No Brasil, a Política Nacional de Promoção da Saúde (PNPS), criada em 2006 e reformulada em 2014, busca a qualidade de vida da população por meio de ações integradas e intersetoriais ao incentivar a participação em conjunto dos setores privados, os governamentais, os não governamentais e a sociedade civil no debate sobre os DSS, potencializando formas ampliadas de intervenção à saúde (Ministério da Saúde, 2014; Malta et al., 2018).

Os DSS podem ser abordados como os aspectos sociais, econômicos, culturais, étnico-raciais, psicológicos, comportamentais e ambientais que intervêm no processo saúde-doença (Ministério da Saúde, 2012a). Para a Comissão Nacional sobre os Determinantes Sociais da Saúde (CNDSS) este conjunto de fatores é amplo e influencia a ocorrência de problemas de saúde e seus aspectos de risco na população (Buss \& Pellegrini Filho, 2007).

A atuação sobre os DSS caracteriza-se por uma composição inter e intrasetorial, sendo que o trabalho da Estratégia Saúde da Família (ESF) destaca-se por ser uma proposta promissora e estruturante na Atenção Primária à Saúde (APS). Entretanto, é necessário organizar uma estrutura de trabalho que ultrapasse os facilitadores individualizados, presentes na prática atual, contemplando aspectos mais amplos (Carrapato, Correia \& Garcia, 2017; Nogueira et al., 2017). 
Urge compreender que uma atenção de qualidade não é alcançada por meio de um cuidado pautado nas ações de um único profissional. É necessário desenvolver uma linha assistencial que contemple a participação da equipe multiprofissional, sendo elaborada coletivamente para alcançar o cuidado integral às gestantes (Schmitz, Heidemann \& Durand, 2018).

É imprescindível trabalhar a clínica ampliada, que inclui os DSS no cotidiano de trabalho das equipes e compete aos profissionais de saúde desenvolver a dimensão cuidadora. Este cuidado demanda a escuta qualificada, o vínculo e o diálogo para além da clínica tradicional, oferecendo respostas efetivas às necessidades da população para atuar de forma integral na assistência à saúde, a fim de evitar as iniquiidades (Arantes, Shimizu \& Merchán-Hamann, 2016).

Nesse âmbito, os profissionais que participam do pré-natal na APS devem levar em conta os aspectos socioeconômicos, culturais e religiosos das gestantes, evitando iniquidades em saúde. Faz-se necessário adentrar na realidade dessas gestantes, buscando articular o conhecimento técnico-científico, na tentativa de intervir e contribuir para um período gestacional sem intercorrências (Schmitz, Heidemann \& Durand, 2018).

Ressalta-se a relevância da atuação sobre os DSS como um caminho para promover a saúde das gestantes. As equipes multiprofissionais necessitam transcender as concepções biológicas e se apropriar dos conceitos de saúde que respondam às diretrizes políticas, os construtos antropológicos, as demandas sociais e a construção histórica, estando cientes de que a saúde é um bem público e encontra-se refém dos DSS que perpassam por macro e micro contextos (Freire, 2019).

Neste estudo emergiu o seguinte questionamento: Como os DSS são trabalhados pela equipe multiprofissional durante o pré-natal na APS? Como objetivo buscou-se compreender como os DSS são trabalhados pela equipe multiprofissional durante o pré-natal na Atenção Primária à Saúde.

\section{Método}

Estudo de abordagem qualitativa, do tipo ação-participante (Pereira et al., 2018). Participaram do estudo 40 profissionais da equipe de Saúde da Família - ESF e do Núcleo Ampliado de Saúde da Família e Atenção Básica (NASF-AB), residentes e acadêmicos da saúde. Os Círculos de Cultura aconteceram em Unidade Básica de Saúde inserida na Atenção Primária de um município do Sul do Brasil.

Como critérios de inclusão elencou-se: ambos os sexos, ser profissional da APS, vinculado à ESF ou NASF-AB, estar presente nos dias de investigação e participar dos CC. Como critério de exclusão adotou-se os profissionais que estavam afastados por férias ou licença no período da investigação. A escolha da UBS seguiu a recomendação da Secretaria Municipal de Saúde, sendo indicada uma que mais atendesse gestantes no seu cotidiano de trabalho. Para garantir o anonimato, os participantes foram identificados por codinomes, nomes de praias.

Como referencial metodológico, utilizou-se do Itinerário de Pesquisa de Paulo Freire, com a utilização do Itinerário de Pesquisa de Paulo Freire, que é constituído por três momentos dialéticos, que estão interligados entre si, sendo eles: investigação temática; codificação e descodificação; e o desvelamento crítico. Estes momentos são concretizados em espaços chamados de Círculo de Cultura (CC), caracterizados por um grupo de pessoas com algum interesse comum que se reúnem para refletir sobre seus problemas, situações de vida, compartilhar vivências, construir uma percepção mais profunda da realidade e estruturar coletivamente ações concretas de intervenção (Heidemann et al., 2017; CNDSS, 2008)

A Figura 1 apresenta estes três momentos dinâmicos e representada pela formação das ondas do mar, conectadas entre si, podendo ocorrer simultaneamente ou não, indo ao encontro das características do território de atuação dos participantes do estudo, que é uma região litorânea. 
Figura 1 - Itinerário de Pesquisa: formação das ondas do mar.

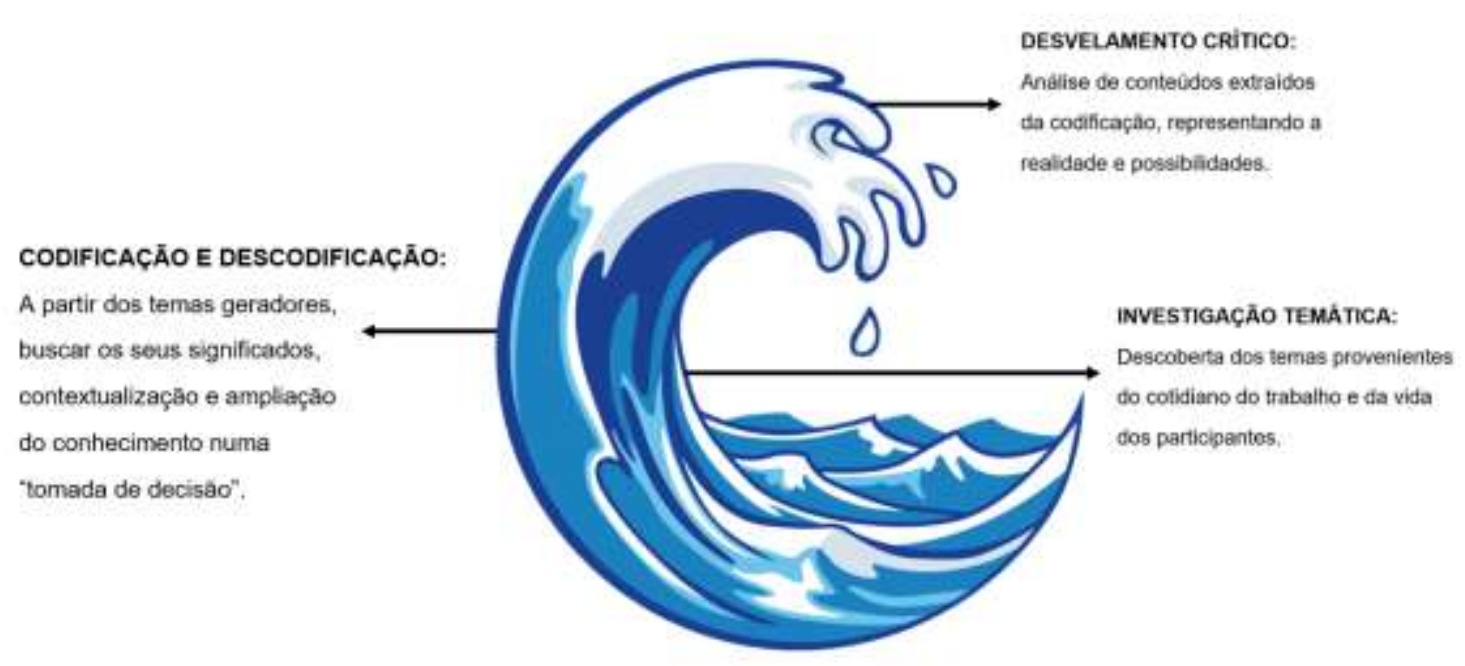

Fonte: Autores.

Foram realizados três CC, com duração aproximada de 90 minutos, entre os meses de julho a outubro de 2019, em uma Unidade Básica de Saúde (UBS) de um município do sul do Brasil. Neste estudo será abordado as discussões realizadas no terceiro CC, em que se dialogou sobre como são trabalhadas as facilidades e dificuldades para abordar os DSS durante o pré-natal. A fim de melhorar a qualidade e a fidelidade dos temas investigados, realizaram-se gravações em áudio com transcrições na íntegra, além de registros fotográficos, durante os Círculos de Cultura.

Para ocorrer a problematização nos CC foi compartilhado com os participantes o modelo de Dahlgren e Whitehead sobre os DSS adotado pela CNDSS, em que são abordados os cinco níveis interdependentes que atuam direta e indiretamente sobre o processo saúde-doença da população (Dalmolin \& Heidemann, 2020).

No primeiro Círculo, os pesquisadores compartilharam o objetivo e a metodologia do estudo. Após iniciou-se a investigação temática a partir da percepção dos profissionais de saúde sobre PS por meio de uma questão disparadora: O que é PS para você? Para provocar a discussão, foi disponibilizado uma tarjeta em branco para que cada participante pudesse escrever ou desenhar sobre o que foi questionado. Após, cada um trouxe a sua reflexão sobre a PS e desta troca formou-se uma mandala construída com as tarjetas. No final os participantes levantaram como temas emergentes: qualidade de vida; tempo e cuidado.

No segundo $\mathrm{CC}$, os pesquisadores resgataram as temáticas investigadas no primeiro encontro e os participantes dialogaram e refletiram, dando início as etapas de codificação, descodificação e desvelamento crítico. Nos diálogos foram destacados 21 temas geradores, que foram codificados em três temas significativos: I) Qualidade de vida; II) Tempo; III) Cuidado.

Os mediadores contextualizaram as temáticas, utilizando analogia com o mar da vida, com participação de todos os envolvidos nos CC. Neste cenário um tecido azul foi estendido ao chão, simbolizando o mar da vida. Os três temas levantados no primeiro $\mathrm{CC}$ foram simbolizados, cada um, por um grande peixe que era acompanhado de peixes menores com os registros dos participantes. Em diálogo, os partícipes foram convidados a olhar para o mar da vida e refletir sobre a mulher gestante, instigando-os a revisitar os fatores que influenciam o bem-estar e a Promoção da Saúde durante o pré-natal, evidenciando os DSS. 
No terceiro CC resgataram-se os temas codificados e descodificados no segundo encontro, sendo apresentados para os participantes em formato de barcos, expostos no mar da vida. Os temas foram divididos em três grupos: I) Socioeconômico, Habitação, Trabalho, Questões alimentares; II) Idade, Sexo, Fatores hereditários, Estilo de vida; III) Família, Rede de apoio, Cultura, Religião. Os partícipes foram divididos em três grupos, e convidados a refletir sobre a vida das gestantes, instigandoos a dialogar sobre como são trabalhados os DSS durante o pré-natal, evidenciando as facilidades e dificuldades para atuação.

Cada grupo escolheu um participante para relatar o que foi discutido, compartilhando suas experiências com o grande grupo, num contínuo processo de ação-reflexão-ação sobre a realidade, descodificando e desvelando o cuidado com a gestante durante o pré-natal. Ressalta-se que, o Círculo despertou a curiosidade dos participantes e permitiu um encontro permeado por profundas reflexões, empoderando-os para discussão da temática, elaborando novos olhares sobre o objeto pesquisado, tomando consciência da realidade (Ribeiro, Aguiar \& Andrade, 2018).

O desvelamento dos temas ocorreu concomitante com a investigação temática, a partir da metodologia de Paulo Freire, que prevê o processo analítico (Heidemann et al., 2017; CNDSS, 2008).

Esta etapa, tradicionalmente chamada de análise dos dados no Itinerário de Pesquisa Freireano, é um processo ininterrupto e ocorre com a interação de todos os participantes que compõem os CC, por meio da leitura, reflexão e interpretação dos temas emergentes. Para facilitar o desvelamento, os temas foram organizados em quadros que expressavam as ideias e temáticas a serem interpretadas.

A pesquisa esteve assegurada nos princípios éticos da resolução 466/12 e 510/16, sendo aprovada pelo Comitê de Ética em Pesquisa da Universidade Federal de Santa Catarina com o parecer 3.253.685 e CAAE 10432719.8.0000.012 de 09 de abril de 2019. A participação na pesquisa foi autorizada mediante a assinatura do Termo de Consentimento Livre e Esclarecido.

\section{Resultados}

O estudo contou com a participação de 40 pessoas, sendo: um médico, quatro enfermeiras, um odontólogo, uma auxiliar de saúde bucal, quatro agentes comunitários de saúde, uma profissional de educação física, um fisioterapeuta, três técnicas de enfermagem, quatro nutricionistas, doze residentes (dois odontólogos, dois nutricionistas, dois médicos, duas enfermeiras, dois profissionais de educação física e dois fisioterapeutas) e oito acadêmicos (dois de nutrição, dois de enfermagem e quatro de medicina). A faixa etária dos participantes variou de 23 a 51 anos e o tempo de atuação na APS variou de um mês a 22 anos.

A seguir serão apresentados os dois desvelamentos que emergiram no CC: I) Desvelando os Determinantes Sociais da Saúde; II) Determinantes Sociais da Saúde: realidade, facilidades e dificuldades.

\section{Desvelando os Determinantes Sociais da Saúde}

Em meio ao movimento de ação-reflexão-ação que aconteceu durante os Círculos de Cultura, desvelou-se importantes aspectos relacionados aos DSS. Os participantes acreditam no potencial dos DSS e percebem a importância de considerá-los durante os atendimentos a população e principalmente na assistência ao pré-natal. Porém foi perceptível que se trata de um tema que necessita ser semeado e regado continuamente para florescer na APS. Nas falas a seguir, pode-se perceber o desvelamento que ocorreu em relação aos DSS.

É muito alegre ouvir falar de Determinantes Sociais, porque os cursos prevalentes nas Universidades são os cursos culpabilizantes e meritocráticos, por isso ouvir isso aqui é muito lindo (Ipanema). 
Eu acho que quando falam sobre essa palavra "Determinantes Sociais da Saúde" que é uma palavra que é acadêmica eu acho que assusta. A primeira vez que eu ouvi, pensei "que coisa chata, lá vem aquela discussão teórica de SUS de novo, meu Deus que coisa chata" (Açores).

A crise política e as mudanças que assolam o Brasil interferem diretamente na saúde, ocasionando preocupações aos profissionais que atuam diretamente na APS, como as dúvidas em relação ao futuro, que por muitas vezes, gera inquietação e revolta e discutir os DSS requer ainda um longo caminho a percorrer.

Fico muito triste, foi liberada uma carteira de serviços ministerial com uma semana para avaliação na qual os DSS não foram levados em consideração e a mulher ainda segue sendo vista como algo reprodutivo. Me entristece! Mas coincidiu de eu estar aqui pensando nos DSS e isto me deixou feliz. Eu estou no lugar certo, com as pessoas certas (Açores)!

Nas reflexões e diálogos durante os círculos de cultura, os participantes refletiram sobre as ações que desenvolvem durante o Pré Natal e perceberam que ao planejarem e executarem estas atividades torna-se possível considerar os fatores determinantes e condicionantes.

Tem um projeto que a enfermeira faz com as gestantes aqui na UBS, fornecendo fotos de estúdios, externas para as gestantes. Percebemos que isso é bem positivo e fortalece o vínculo da família. É aquele momento do casal, às vezes o casal já tem mais filho e, os filhos participam também... é um momento de interação, de fortalecimento (Ponta das Canas).

Nos encontros trabalhamos também... sobre os direitos da gestante que é algo importante, conversamos sobre a questão da renda e sobre os direitos trabalhistas (Praia do Rosa).

\section{Determinantes Sociais da Saúde: realidade, facilidades e dificuldades}

Os participantes dialogaram sobre a importância da família durante o pré-natal. Identificar a rede de apoio e inclui-la no pré-natal é visto como algo fortalecedor pelos profissionais. Segundo eles, as gestantes que não possuem uma rede de apoio fortalecida, apresentam maior dificuldade para lidar com as questões que envolvem o processo de gestar.

Em relação à rede de apoio, identificar a que existe...durante a consulta e direcionamos. A maior dificuldade são as gestantes que vem morar aqui na cidade e não possuem amigos, parentes, os avós moram longe, não conseguem estar presentes para auxiliar. A mulher muitas vezes não tem o companheiro/parceiro para auxiliar (Lagoinha do Leste).

Em relação à família no pré-natal, a gente busca incluir, principalmente os parceiros, até porque são as pessoas que mais acompanham as gestantes, eventualmente são as avós ou os filhos mais velhos (Lagoinha do Leste).

Também tem a carteira de pré-natal, que tem a parte de pré-natal do parceiro. Nós procuramos usar isto como uma forma de chamar o esposo/companheiro para estar presente, oferecendo cuidado para ele, incluindo-o (Ponta das Canas). 
Reflexões sobre escuta qualificada e empatia vieram à tona durante os diálogos nos Círculos de Cultura. O acolhimento realizado de forma adequada, prezando a equidade e um plano de cuidados que considere os fatores como idade e estilo de vida, são exemplos de ações consideradas importantes pelos profissionais.

A gente tem a escuta qualificada, a empatia, acolhemos bem as nossas gestantes, tanto nas consultas, quanto no grupo de gestantes (Galheta).

Seguimos o princípio de SUS de equidade, que é tratar diferente os diferentes, levando todos esses determinantes sexo, fatores hereditários e estilo de vida em consideração. Cada gestante é diferente e a gente tem que levar isso em conta, é diferente uma consulta de uma menina de 19 anos, esperando o primeiro filho, nós temos que repassar muito mais orientações para ela comparando com uma mulher de 35 anos, que já tem três filhos (Pântano do Sul).

Além da idade, tem o fator que é o estilo de vida, cada uma tem o seu estilo de vida (Galheta).

A cultura foi um dos fatores citados como dificuldade para trabalhar os DSS. Os profissionais relataram que comportamentos culturais podem interferir no cuidado pré-natal. As ações populares, realizadas principalmente pelas avós e mães das gestantes também são percebidas como uma dificuldade.

Em relação à cultura, a gente identifica como uma dificuldade, a exemplo, constrangimento no atendimento. Porque às vezes pode acontecer de ser atendida por um homem e dependendo do assunto que vai ser tratado, como uma questão sexual, uma hemorroida, alguma coisa que exista algum exame ginecológico, pode se encaminhar o atendimento em conjunto ou com uma mulher. Existe a dificuldade cultural de encontrar uma evidência científica que corrobore com as orientações das avós em determinadas coisas e isso necessita de uma competência cultural, para aproximar as orientações populares/culturais das orientações científicas (...) (Illha do Campeche).

Os participantes dialogaram também que o tempo que possuem para a consulta de pré-natal não é o ideal, pois a demanda excessiva e a falta de recursos humanos limitam o tempo das consultas e deveria ser abordado as questões sociais na consulta com as gestantes.

Uma dificuldade que a gente possui é falta de tempo para a consulta, muitas vezes não temos 01h ou 01h30min para a consulta, e falta também profissionais e capacitação continuada (Galheta).

O trabalho em equipe com a abordagem multiprofissional e intersetorial supre muitas vezes, a demanda social da APS e a gestante possui prioridade no atendimento com o NASF-AB. No entanto, os partícipes revelaram que há dificuldades de acesso, acolhimento, assistência a gestante que requerem avanços e resolutividade nos serviços de saúde. Os profissionais destacaram que a falta de governabilidade sobre alguns DSS dificulta o desenvolvimento do pré-natal.

Percebe-se situações de assédio trabalhista em relação às gestantes. Geralmente encaminhamos para a equipe de apoio, a assistente social, a psicóloga ajuda, apesar da restrição de vagas por limite da carga horário da profissional (Ilha do Campeche).

A falta de governabilidade sobre algumas situações é uma dificuldade, principalmente a renda, habitação precária, alimentação (Praia do Rosa). 
Os profissionais da APS percebem a importância dos DSS e compreendem que existem obstáculos para além dos muros da UBS que precisam ser enfrentadas.

\section{Discussão}

A PS em sinergia com os DSS, podem contribuir para uma melhor compreensão e endereçamento das iniquidades de saúde e alcance da equidade (Ministério da Saúde, 2014). Portanto, espera-se que a PS tenha como foco a ação sanitarista, atuando em conjunto com os DSS, que incluem: o próprio indivíduo e seu estilo de vida; as redes sociais e comunitárias; a oferta de serviços como saúde, educação, habitação e saneamento; além das condições socioeconômicas, culturais e ambientais (Theophilo, Rattner \& Pereira, 2018).

A redução das iniquidades depende do conjunto de ações dirigidas à garantia do direito à saúde em todas as suas dimensões, incluindo a melhoria dos determinantes e condicionantes da saúde. Desta forma, necessita-se formular e implementar políticas públicas que visem a redução destas iniquidades (Dalmolin, Heidemann \& Freitag, 2019). No entanto, apesar da clareza sobre a importância da implementação de políticas públicas que garantam a eficácia e a eficiência do sistema de saúde, a perspectiva dos DSS aponta que os modelos assistenciais de cuidado em saúde não são suficientes para efetivamente melhorar a saúde individual e da população, bem como promover a equidade em saúde (Theophilo, Rattner \& Pereira, 2018).

Foi perceptível durante os diálogos e reflexões que os participantes reconhecem os DSS e sabem da importância de inseri-los na atuação da APS, principalmente tratando-se do cuidado pré-natal. Não obstante, desvelou-se ser um tema que precisa ser aprofundado entre os profissionais que atuam na APS, por meio da educação continuada. Somado a isso, ficou evidente que, além da formação e atuação dos profissionais da saúde, emerge também a necessidade de se ater às realidades da população, levando em conta os fatores sociais, culturais, as crenças e os valores, em busca de unir o saber científico ao saber popular do indivíduo, com foco na valorização da subjetividade do cuidado (Garbois, Sodré \& Dalbello-Araújo, 2017).

A atuação sobre os DSS abrange as parcerias intersetoriais, por meio de serviços que influenciam diretamente a qualidade de vida da população. Entretanto, essas parcerias ainda estão muito focadas na escola, no conselho tutelar, lares de idosos e organizações não governamentais, que são setores diretamente ligados às atividades de saúde, enquanto deveria abranger a intersetorialidade de modo amplo, ultrapassando os limites das unidades sanitárias (Ministério da Saúde, 2014).

Do ponto de vista das políticas públicas de saúde, entende-se que insistir na separação entre o social e a saúde significa contrariar o princípio da integralidade, o que reforça a fragmentação da vida, construindo atalhos contrários às diretrizes do SUS. As questões sociais não podem ser vistas como 'causas externas' ao setor saúde, pois reduz a capacidade política de todos os atores sociais para agir em busca da sua qualidade de vida. Na perspectiva das práticas cotidianas de atenção à saúde, considerando o pressuposto que todo ato em saúde é dotado de significação, adotar a noção de DSS reforça a polaridade estabelecida entre o ser biológico e o ser social. É nessa lógica que o sujeito é considerado como aquele que sofre violência familiar, que vive em condições precárias de vida e de trabalho, que depende das políticas de assistência social (Gaíva, Palmeira \& Mufato, 2017).

Destaca-se a equipe multiprofissional como uma fortaleza para a atuação sobre os DSS, considerando a realidade das gestantes e articulando com o conhecimento técnico-científico. Compreende-se que uma atenção de qualidade não é alcançada por meio de um cuidado pautado nas ações de um único profissional. Torna-se necessário desenvolver uma linha assistencial que contemple a participação de toda a equipe, sendo elaborada de maneira coletiva para que, de fato, ocorra o cuidado integral às gestantes (Schmitz, Heidemann \& Durand, 2018). 
O cuidado integral gera qualificação na assistência durante o pré-natal, sendo vinculado aos profissionais que dispõem da escuta qualificada, que dialogam sobre a gestação e os cuidados obstétricos e neonatais, aos que se disponibilizam a sanar dúvidas e acolher, mesmo fora das consultas mensais obrigatórias, escutando os anseios destas mulheres e de suas famílias (Romagnolo et al., 2017).

Além do trabalho em equipe, a importância da família foi evidenciada durante o estudo como algo fortalecedor durante o pré-natal. O tornar-se mãe é uma das transições importantes e difíceis da vida adulta. A gravidez e o puerpério são períodos cruciais, tanto biológicos, quanto psicológicos devido às alterações fisiológicas que preparam a gestante para o parto, nascimento e cuidados posteriores. Nesta fase, torna-se imprescindível a revisão dos papéis sociais e familiares (Prates, Schmalfuss \& Lipinski, 2015). Percebe-se que, além de oferecer suporte, apoio, proporciona exemplo de como viver, amar, sentir, cuidar de si e cuidar do outro, representando o agente socializador primário do indivíduo. Dentro do sistema familiar o sujeito conecta-se a crenças, valores e tradições que estão diretamente relacionados com os cuidados em saúde. As ligações familiares possuem a capacidade de influenciar a forma com a qual os indivíduos percebem e vivenciam o processo saúdedoença e o cuidado com seus membros, sendo perceptível tal cuidado durante o período de gestação, parto e pós-parto (Urbanetto et al., 2017).

Em relação às dificuldades na atuação sobre os DSS relacionadas a cultura, saber popular vivenciado durante a gestação, parto e puerpério, instiga-se este momento como um fenômeno complexo e singular. O profissional de saúde deve trabalhar com as gestantes, respeitando seus valores e crenças. Ressalta-se a importância de as equipes de saúde reconhecerem a íntima relação entre mãe e filho e utilizar da imponência das avós como auxílio durante o pré-natal e puerpério (Leonello, Vieira \& Duarte, 2018). Além disso, uma equipe de saúde, quando insuficiente em número e qualificação, pode influenciar de forma negativa a prestação dos cuidados às famílias, podendo desencadear um fator gerador de negligência nas ações de saúde.

\section{Conclusão}

A atuação sobre os DSS na assistência a gestante faz parte da realidade atual dos partícipes, apesar de muitas vezes os profissionais não compreenderem de modo amplo o seu conceito. Ao refletirem como essa atuação acontece, emergiram experiências exitosas com a abordagem nos DSS, como os grupos de gestantes e os projetos envolvendo as famílias.

A escuta qualificada e a empatia também foram desveladas durante os CC. Os profissionais da APS consideram alguns fatores relacionados aos DSS, como idade e estilo de vida e procuram entender a realidade em que estas mulheres estão inseridas para elaborar um plano de cuidados resolutivo para a gestante e sua família. Como fator limitante em relação à atuação sobre os DSS foi possível perceber que a falta de recursos humanos, perante a demanda excessiva de usuários da APS é algo que diminui a qualidade da assistência prestada, apesar da atuação conjunta da equipe multiprofissional.

Os profissionais buscam compreender as questões sociais, todavia a atuação sobre os DSS acontece limitadas ao setor saúde. Percebeu-se que os fatores como trabalho, renda e moradia são as questões que mais preocupam em relação ao pré-natal, especialmente por se tratarem de aspectos que os participantes têm pouca ou nenhuma governabilidade.

O método escolhido oportunizou momentos de reflexão e compartilhamento de saberes entre os participantes e as pesquisadoras, tornando os $\mathrm{CC}$ espaços acolhedores e reflexivos, contribuindo para a melhoria do processo de trabalho na APS. Destaca-se, porém, como limitações o tempo restrito para a realização dos CC, que foram desenvolvidos durante as reuniões da eSF, de modo a promover a participação dos profissionais da APS.

Ressalta-se ainda o número limitado de estudos no âmbito dos DSS no pré-natal. Sugere-se a realização de novos estudos, com o intuito de desvelar e ampliar a atuação dos DSS na APS, principalmente no pré-natal, por ser um momento que envolve não somente o binômio mãe-bebê, mas toda estrutura familiar, trazendo demandas sociais que precisam ser consideradas para o alcance da PS. 


\section{Agradecimentos}

Conselho Nacional de Pesquisa e Desenvolvimento - CNPq.

\section{Referências}

Arantes, L. J., Shimizu, H. E., \& Merchán-Hamann, E. (2016). Contribuições e desafios da Estratégia Saúde da Família na Atenção Primária à Saúde no Brasil: revisão da literatura. Ciência \& Saúde Coletiva, 21(5), 1499-1510. https://www.scielo.br/scielo.php?pid=S1413-81232016000501499\& script=sci_abstract\&tlng=pt

Buss, P.M. (2010). O conceito de promoção da saúde e os determinantes sociais. Ecodebate.

Buss, P. M., \& Pellegrini Filho, A. (2007). A Saúde e seus determinantes sociais. Physis, 1(17), 77-93. https://www.scielo.br/pdf/physis/v17n1/v17n1a06.pdf

Carrapato, P., Correia, P., \& Garcia, B. (2017). Determinante da saúde no Brasil: a procura da equidade na saúde. Saúde e Sociedade, 26(3), 676-689. https://www.scielo.br/scielo.php?script=sci_abstract\&pid=S0104-12902017000300676\&lng=pt\&nrm=iso\&tlng=pt

CNDSS. (2008). As causas sociais das iniquidades em saúde no Brasil. Editora FIOCRUZ. http://bvsms.saude.gov.br/bvs/publicacoes/ca usas_sociais_iniquidades.pdf

Costa, J. C., Nitschke, R. G., Rocha, P. F. A. \& Andrade, B. B. (2019). O imaginário da promoção da saúde no quotidiano das famílias na atenção primária. Revista de Enfermagem da UFSM, 9(65), 01-19. https://periodicos.ufsm.br/reufsm/article/view/33603

Dalmolin, I. S. \& Heidemann, I. T. S. B. (2020). Práticas integrativas e complementares na Atenção Primária: desvelando a promoção da saúde. Rev. LatinoAm. Enfermagem, 28, e3277. https://doi.org/10.1590/1518-8345.3162.3277.

Dalmolin, I. S., Heidemann, I. T. S. B. \& Freitag, V. L. (2019). Práticas integrativas e complementares no Sistema Único de Saúde: desvelando potências e limites. Rev Esc Enferm USP, 53, e03506. https://www.scielo.br/pdf/reeusp/v53/1980-220X-reeusp-53-e03506.pdf

Freire, P. (2019). Pedagogia do Oprimido. (75a ed.), Paz e Terra.

Gaíva, M. A. M., Palmeira, E. W. M., \& Mufato, L. F. (2017). Women'sperception of prenatal and delivery care in cases of neonatal death. Esc. Anna Nery Rev. Enferm., 21(4). e20170018. https://www.scielo.br/pdf/ean/v21n4/1414-8145-ean-2177-9465-EAN-2017-0018.pdf

Garbois, J. Á., Sodré, F., \& Dalbello-Araujo, M. (2017). Da noção de determinação social à determinantes sociais da saúde. Saúde em Debate., 41(112). 63-76. https://www.scielosp.org/pdf/sdeb/2017.v41n112/63-76

Heidemann, I. T. S. B., Almeida, M. C. P., Boehs, A. E., Wosny, A. M. \& Monticelli, M. (2006). Promoção à saúde: trajetória histórica de suas concepções. Texto contexto-enferm., 15(2), 352-358. https://www.scielo.br/scielo.php?pid=S0104-07072006000200021\&script=sci_abstract\&tlng=pt

Heidemann, I. T. S. B., Cypriano, C. C., Gastaldo, D., Jackson, S., Rocha, C. G. \& Fagundes, E. (2018). Estudo comparativo de práticas de promoção da saúde na atenção primária em Florianópolis, Santa Catarina, Brasil e Toronto, Ontário, Canadá. Cad. Saúde Pública., 34(4), 1-13. https://www.scielo.br/pdf/csp/v34n4/1678-4464-csp-34-04-e00214516.pdf

Heidemann, I. T. S. B., Sartori, I. D., Rumor, P. C. M., Costa, C. C., Costa, M. F. B. A. \& Durand, M. K. (2017). Reflexões sobre o Itinerário de pesquisa de Paulo Freire: contribuições para a saúde. Texto contexto - enferm, 26(4). 1-8. https://www.scielo.br/pdf/tce/v26n4/0104-0707-tce-26-04-e0680017.pdf

Leonello, V. M., Vieira M. P. M. \& Duarte, T. C. R. (2018). Competencies for education al actions of Family Health Strategy nurses. Rev Bras Enferm., 71(3). 1072-78. https://www.scielo.br/scielo.php?pid=S0034-71672018000301072\&script=sci_arttext

Malta, D. C., Reis, A. A. C., Jaime, P. C., Neto, O. L M., Silva, M. M. A. \& Akerman, M. O. (2018). SUS e a Política Nacional de Promoção da Saúde: perspectiva resultados, avanços e desafios em tempos de crise. Ciência \& Saúde Coletiva., 23(6). 1799-1809. https://www.scielo.br/scielo.php?pid=S1413$81232018000601799 \&$ script=sci_arttext

Ministério da Saúde (Brasil). (2012). Secretaria de Vigilância em Saúde. Glossário temático: promoção da saúde. Brasília.

Ministério da Saúde (Brasil). (2014). Secretaria de Atenção à Saúde. Política Nacional de Promoção da Saúde. Brasília-DF.

Nogueira, C. M. C. S., Sousa, C. N. S., Nóbrega, L. L. R., Sales, L. K. O., \& Morais, F. R. R. (2017). Assistência ao pré-natal e as práticas desenvolvidas pela equipe de saúde: revisão integrativa. Revista de Pesquisa: Cuidado é Fundamental Online, 9(1). 279-288. http://www.seer.unirio.br/index.php/cu idadofundamental/article/view/4184

Pereira, A. S. et al. (2018). Metodologia da pesquisa científica. UFSM. https://repositorio.ufsm.br/bitstream/handle/1/15824/Lic_Computacao_MetodologiaPesquisa-Cientifica.pdf?sequence $=1$

Pinheiro, D. G. M., Scabar, T. G., Maeda, S. T., Fracolli, L. A., Pelicioni, M. C. F. \& Chiesa, A. M. (2015). Competências em promoção da saúde: desafios da formação. Saúde soc., 24(1), 180-188. https://www.redalyc.org/articulo.oa?id=406263640023

Prates, L. A., Schmalfuss, J. M. \& Lipinski, J. M. (2015). Social support network of post-partum mothers in the practice of breastfeeding. Esc. Anna Nery Rev. Enferm., 19(2). 310-315. https://www.scielo.br/pdf/ean/v19n2/en_1414-8145-ean-19-02-0310.pdf

Ribeiro, K. G., Aguiar, J. B. \& Andrade, L. O. M. (2018). Determinantes sociais da saúde: o instituído constitucional no Sistema Único de Saúde. Ver Bras Promoç Saúde., 31(4), 01-10. https://periodicos.unifor.br/RBPS/article/view/8778 
Research, Society and Development, v. 10, n. 3, e50510313434, 2021

(CC BY 4.0) | ISSN 2525-3409 | DOI: http://dx.doi.org/10.33448/rsd-v10i3.13434

Romagnolo, A. N., Costa, A. O., Souza, N. L., Somera, V. C. O. \& Gomes, M. B. (2017). A família como fator de risco e de proteção na gestação, parto e pósparto. Semina Ciênc. Soc. Hum., 38(2). 133-146. http://www.uel.br/revistas/uel/index.php/seminasoc/article/view/31412

Schmitz, C. C. C., Heidemann, I. T. S. B. \& Durand, M. K. (2018). A Atuação dos Profissionais da Atenção Primária Acerca das Práticas de Promoção e dos Determinantes Sociais da Saúde. Revista Enfermagem Atual In Derme., 86(24). 1-13. https://revistaenfermagematual.com.br/index.php/revista/article/view/92

Theophilo, R. L., Rattner, D. \& Pereira E, L. (2018). Vulnerabilidade de mulheres negras na atenção ao pré-natal e ao parto no SUS: análise da pesquisa da Ouvidoria Ativa. Ciênc. saúde coletiva, 23(11). 3505-17. https://www.scielo.br/scielo.php?script=sci_abstract\&pid=S1413$81232018001103505 \& \operatorname{lng}=$ pt\&nrm=iso

Urbanetto, P. D. G., Gomes, G. C., Costa, A. R., Nobre, C. M. G. N., Xavier, D. M. \& Silva, J. G. (2017). Orientações recebidas pelas gestantes no pré-natal acerca da amamentação. Ciênc. saúde coletiva, 16(4). 1-8. http://periodicos.uem.br/ojs/index.php/CiencCuidSaude/article/view/34071/22008

World Health Organization. (1986). Ottawa Charter for Health Promotion. Ottawa: World Health Organization, Health and Welfare Canada, Canada Public Health Association. https://www.who.int/teams/health-promotion/enhanced-wellbeing/first-global-conference 